\title{
Eosinophilic Pneumonia Associated with Rheumatoid Arthritis: Description of a Case Report and Review of the Literature
}

\author{
Marta Priora ${ }^{1}$, Lorenzo Vassallo ${ }^{2}$, Marco Scarati ${ }^{1}$, Simone Parisi $^{1}$, Enrico Fusaro $^{1}$ \\ ${ }^{1}$ Rheumatology Department, AOU Città della Salute e della Scienza di Torino, Italy \\ ${ }^{2}$ Radiology Department, AOU Città della Salute e della Scienza di Torino, Italy
}

Received: $15 / 06 / 2015$

Accepted: $23 / 08 / 2015$

Published: 18/09/2015

\begin{abstract}
How to cite this article: Priora M, Vassallo L, Scarati M, Parisi S, Fusaro F. Eosinophilic pneumonia associated with rheumatoid arthritis: description of a case report and review of the literature. EJCRIM 2015;2:doi:10.12890/2015_000241
\end{abstract}

Conflicts of Interests: The authors declare that there are no competing interests.

Patient's Consent: The authors declare they obtained patient's consent for publication.

This article is licensed under a Commons Attribution Non-Commercial 4.0 License

\section{ABSTRACT}

Rheumatoid arthritis, a systemic inflammatory disease, may induce pulmonary manifestations. We describe a case of longstanding RA presenting with eosinophilic pneumonia. Rare case reports of tissue eosinophilia involving isolated organs in the setting of rheumatoid arthritis exist in the literature. It has been shown that the production of proinflammatory cytokines activates different cell group and can simultaneously play a role in rheumatoid arthritis, and induce eosinophils infiltration in target tissue. An appropriate lowest possible dosage of steroid therapy is essential, whereas eosinophilic pneumonia may be a rare subset of pulmonary involvement in rheumatoid arthritis.

\section{LEARNING POINTS}

- The lungs and pleura are frequently involved in rheumatoid arthritis and contribute between $10 \%$ and $20 \%$ of overall mortality.

- A patient with rheumatoid arthritis presenting with respiratory symptoms, serum eosinophilia and specific pulmonary radiological involvement, can in rare cases have eosinophilic pneumonia.

- There may be an association between hypereosinophilia and immunological alteration in rheumatoid arthritis.

- In this patient, treatment with prednisone and hydroxychloroquine achieved clinical remission.

\section{KEYWORDS}

Eosinophilic pneumonia, rheumatoid arthritis, steroid therapy.

\section{INTRODUCTION}

Rheumatoid arthritis (RA) is a systemic inflammatory disease that primarily affects the synovial joints with a chronic progressive erosive synovitis which leads to destruction of both bone and cartilage. Multiple other organs may be involved, with extra-articular manifestations in the cardiovascular system, lungs, skin and eyes. The lungs and pleura are frequently involved and contribute between $10 \%$ and $20 \%$ of overall mortality. Recent pathogenetic findings provide fascinating insights into how pneumopathies may be related to the development of $\mathrm{RA}^{[1,2]}$. Pulmonary parenchymal lesions found in RA are classified into different histopathological subtypes. Eosinophilic pneumonia (EP) in RA is a clinical entity so uncommon it is rarely seen. Some author ${ }^{[3,4]}$ documented a hypereosinophilia in the serum of up to $40 \%$ of patients with RA during the active phase of the disease. Other authors ${ }^{[5-8]}$ support the hypothesis that pathological activation of eosinophils may correlate with the presence of rheumatoid factor (RF) since a higher incidence of eosinophilic tissue infiltration was found in patients with eosinophilia and high serum RF. 


\section{CASE REPORT}

A 61-year-old Italian man, who used to smoke until he was 30 and with no known allergies, was admitted to our rheumatology department in 1990 for rapid progressive onset of symmetric arthritis, with swelling and tenderness on both the metacarpophalangeal and proximal interphalangeal joints of his hands and on the bilateral metatarsophalangeal joints of his feet. He also presented with considerable morning stiffness, significantly increased inflammatory markers and RF positivity. X-rays showed no signs of bone erosion. The patient was diagnosed with early-stage rheumatoid arthritis (RA) according to the 1987 American College of Rheumatology (ACR) criteria (the diagnosis would also be supported by the latest 2010 ACR criteria). Treatment with hydroxychloroquine (HCQ) 200 mg/day plus prednisone 5 mg/day produced an excellent clinical response. In February 2010, the patient had an episode of acute idiopathic (not lithiasic or alcoholic) pancreatitis. During hospitalization, radiological abdominal investigations were negative and the only pathological finding was constant leucocytosis with hypereosinophilia in blood tests, with average values of $22.3 \%$ of eosinophils (normal maximum $4 \%$ ), with a mean absolute value of

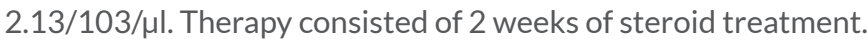

Continuing light therapy with HCQ prevented clinical, serological or radiological exacerbation or progression of the RA, with RF constantly positive with a medium titre of $44 \mathrm{IU} / \mathrm{ml}$ (normal value $20 \mathrm{IU} / \mathrm{ml}$ ) and negative anti-citrullinated peptide antibodies (ACPA). Prednisone was suspended in April 2011. However, 4 months later, the patient was admitted to hospital with cough and high fever. Blood tests showed that eosinophils were consistently high with an average value of $21.2 \%$ (absolute value of 2.40/109/I). Chest x-rays showed extensive pulmonary parenchymal bilateral consolidations, mainly in the right upper lobe (Fig. 1). Computed tomography (CT) of the chest confirmed the presence of extensive bilateral nodular parenchymal infiltrates (Fig. 2). Bronchoscopy, cytology of bronchoalveolar fluid, cultures and virological tests were negative for tumours and infections, but the bronchoalveolar fluid demonstrated a high eosinophilic count (35\%). Neither existing nor previous nasal polyposis or peripheral neuropathy were found. RF was $378 \mathrm{mg} / \mathrm{dl}$, while anti-nuclear antibodies, extractable nuclear antigens, anti-dsDNA, anti-neutrophil cytoplasmic antibodies and ACPA antibodies were negative. Pulmonary function tests were normal..
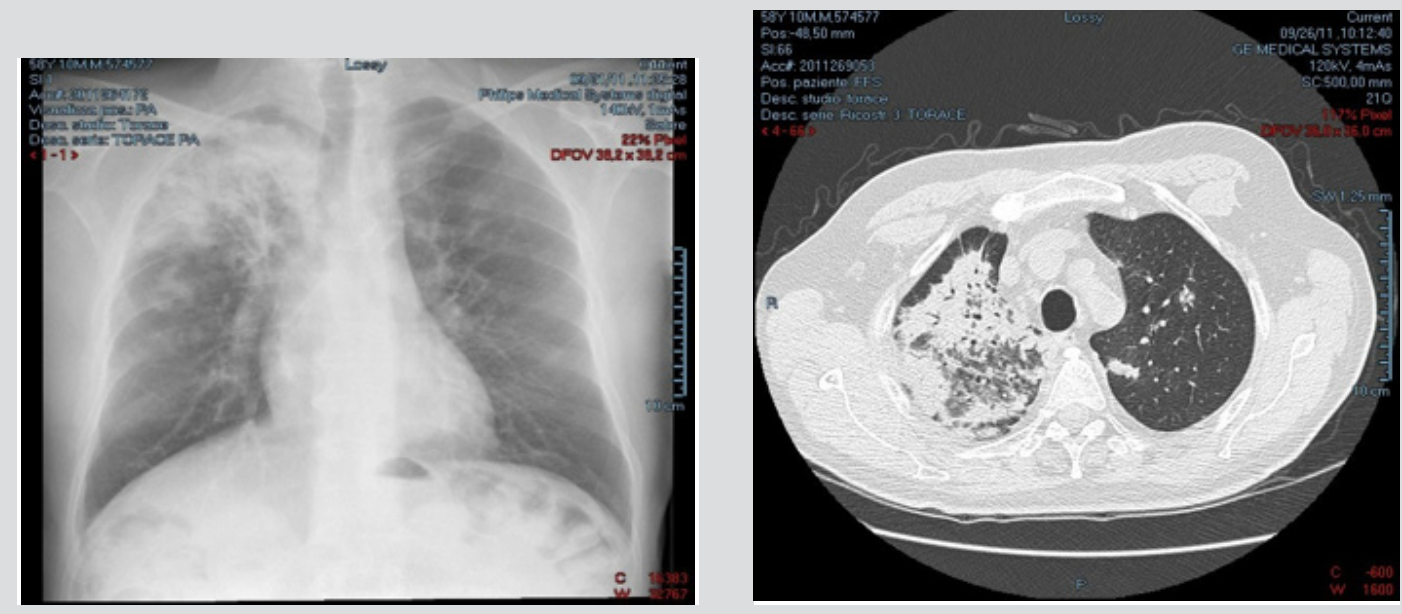

Figure 1: Chest $X$-rays of the patient in 2011, at the time of diagnosis of Eosinophilic Pneumonia, showing pulmonary parenchymal bilateral consolidations, mainly at the right upper lobe.

Figure 2: Computes Tomography of the chest, dating in 2011, documenting the presence of bilateral parenchymal infiltrates of the lungs.

Transbronchial lung biopsy crucially demonstrated "fragments of bronchial wall with fibrosis and intense infiltrate of eosinophils and frustules of lung parenchyma with lympho-granulocyte inflammation with a rich eosinophilic interstitial component". The diagnosis of eosinophilic pneumonia (EP) was followed by therapy consisting of high doses of steroids with rapid regression of clinical and radiological signs.

In October 2012, after a gradual tapering of steroids, a recurrence of the same pulmonary pattern of EP required that therapy be restarted. The reduction in prednisone to under $10 \mathrm{mg} /$ day had caused the reappearance on CT of bilateral nodular consolidations, mainly in the upper lobes.

Currently, the patient is asymptomatic and continues steroid treatment with prednisone (10 mg/day) and HCQ. Laboratory and clinical values are satisfactory, with constant RF positivity (372 IU/ml in November 2013) and ACPA negativity. Structural deformation of the hands is shown in Figs. 3, 4 and 5. Chest $x$-rays and high-resolution CT are currently negative. 


\section{DISCUSSION}

In the setting of a hypereosinophilic state in RA (excluding allergic, infectious and drug-related disease), case reports of tissue eosinophilia involving isolated organs are rare in the literature, but the possibility of such an association between these haematological and rheumatological entities has been discussed ${ }^{[9-11]}$.

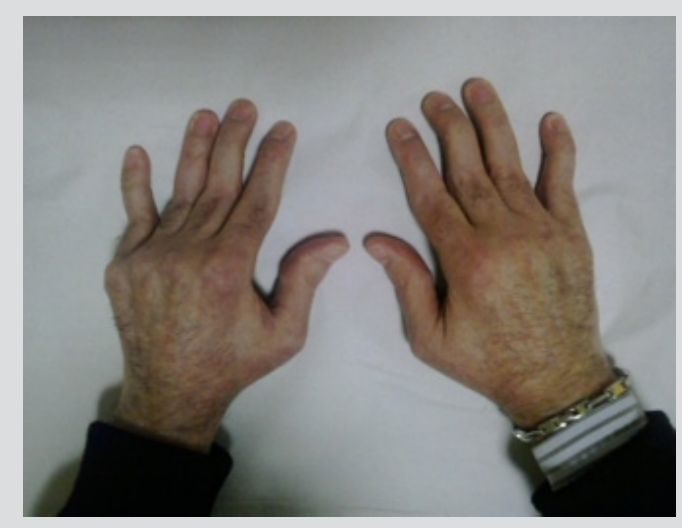

Figure 3: Morphological deformations of hands that currently presents the patient.
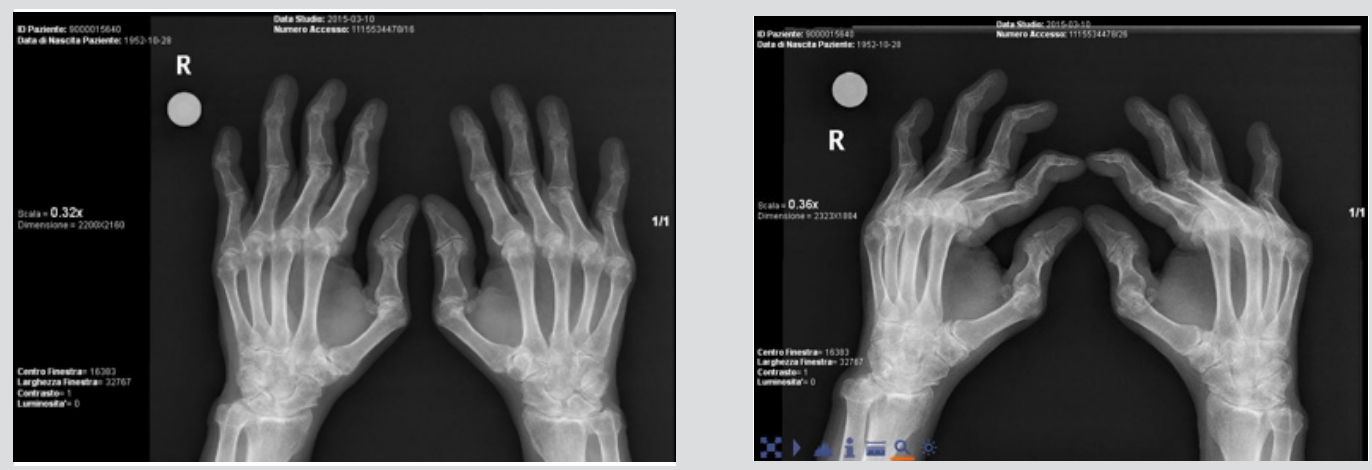

Figure 4: $X$-ray image of the hands (posteroanterior view) showing articular deformation presented by the patient nowadays.

Figure 5: $X$-ray image of the hands (oblique view) showing articular deformation presented by the patient nowadays.

The first description of EP associated with RA was provided by Payne et al. ${ }^{[12]}$, who sought to demonstrate a common origin unrelated to autoimmunity. Later in 1985 Yousem et al. ${ }^{[3]}$ histologically analysed the lungs of 40 randomly chosen patients with RA and associated pulmonary involvement: one of the 40 had an EP. Review of the literature does not reveal a correlation between the severity of EP and the time of diagnosis of RA, the patient's age, sex or ACPA positivity.

In 2001 Van Esch et al. ${ }^{[13]}$ described how (hyper)activation of T cells, through cross-linking with B cells mediated by CD40, is responsible simultaneously for systemic eosinophil chemotaxis and the production of RF.

More recently, the role of some interleukins (IL6, IL5, IL10) and chemokines (eotaxines 1 and 2) has been shown to induce eosinophil recruitment by macrophages and T lymphocytes and inhibit apoptosis of eosinophilic infiltrates in tissue.

As evidenced in animal studies ${ }^{[14]}$, the mechanisms inducing eosinophilic recruitment are mainly driven by a lymphocyte-TH2 pathway. However, RA is a predominantly TH1/TH17-driven disease, while TH2-cytokines can have an ameliorating effect.

Sandhya et al. ${ }^{[15]}$ suggest that the switch from a TH1/TH17 to a TH2 immunological pathway may be the mechanism which both causes RA remission and induces the release of eosinophils into the blood stream, with consequent infiltration in specific target organs ${ }^{[16-18]}$. As shown by the data, our patient as well as those reported by other authors, had inactive RA at the time of diagnosis of eosinophilia[19].

\section{CONCLUSION}

The clinical picture of a patient with longstanding inactive deforming RA and presenting with respiratory symptoms, serum eosinophilia and specific pulmonary radiological involvement, could lead to a diagnosis of EP in rare cases. During the diagnostic pathway, drug-induced reactions, other interstitial pulmonary diseases as well as parasitic and viral infections have to be excluded as a first step. 
Appropriate steroid treatment is essential. Our patient was maintained in clinical and radiological remission with prednisone $10 \mathrm{mg} / \mathrm{day}$ and HCQ 200 mg/day. Any reduction in steroid dosage, and of course its suspension, caused clinical, immunological and radiological reactivation.

\section{REFERENCES}

1. Antin-Ozerkis D, Evans J, Rubinowitz A, Homer RJ, Matthay RA. Pulmonary manifestations of rheumatoid arthritis. Clin Chest Med 2010;31:451-478.

2. Lake F, Proudman S. Rheumatoid arthritis and lung disease: from mechanisms to a practical approach. Semin Respir Crit Care Med 2014;35:222-238.

3. Yousem SA, Colby TV, Carrington CB. Lung biopsy in rheumatoid arthritis. Am Rev Respir Dis 1985;131:770-777.

4. Winchester RJ, Koffler D, Litwin SD, Kurikel HG. Observation on the eosinophilia of certain patients with rheumatoid arthritis. Arthritis Rheum 1971;14:650-665.

5. Kudou M, Yasuba H, Kobayashi Y, Hamada K, Kita H. Correlation between rheumatoid factor and peripheral eosinophil count in chronic eosinophilic pneumonia. Respirology 2006;11:830-832.

6. Jaimes-Hernandez J, Mendoza-Fuentes A, Meléndez-Mercado $\mathrm{Cl}$, Aranda-Pereira P. Chronic eosinophilic pneumonia: autoimmune phenomenon or immunoallergic disease? Case report and literature review. Reumatol Clin 2012;8:145-148.

7. Boudou L, Alexandre C, Thomas T, Pallot-Prade B. Chronic eosinophilic pneumonia (Carrington's disease) and rheumatoid arthritis. Joint Bone Spine 2010;77:477-480.

8. Tampo Y, Fujimura M, Yasui M, Kasahara K, Nakatsumi Y, Nakao S. Eosinophilic pneumonia (EP) associated with rheumatoid arthritis in which drug-induced eosinophilic pneumonia could be ruled out. Intern Med 2008;47:527-531.

9. Kwak JJ, Chang JE, Lee J, Cho YJ, Sung SH. Chronic eosinophilic pneumonia associated with an initiation of rheumatoid arthritis. Clin Rheumatol 2003;22:240-243.

10. Norman D, Piecyk M, Roberts DH. Eosinophilic pneumonia as an initial manifestation of rheumatoid arthritis. Chest 2004;126:993-995.

11. Hallgren R, Feltelius N, Svenson K, Venge P. Eosinophil involvement in rheumatoid arthritis as reflected by elevated serum levels of eosinophil cationic protein. Clin Exp Immunol 1985;59:539-546.

12. Payne CR, Connellan SJ. Chronic eosinophilic pneumonia complicating long-standing rheumatoid arthritis. Postgrad Med J 1980;56:519-520.

13. Van Esch WJ, Reparon-Schuijt CC, Levarht EW, Van Kooten C, Breedveld FC, Verweij CL. Differential requirements for induction of total immunoglobulin and physiological rheumatoid factor production by human peripheral blood B cells. Clin Exp Immunol 2001;123:496-504.

14. Lucendo AJ. Eosinophilic diseases of the gastrointestinal tract. Scand J Gastroenterol 2010;45:1013-1021.

15. Sandhya P, Danda D, Mathew J, Kurian S, Ramakrishna BS. Eosinophilic esophagitis and pharyngitis presenting as mass lesion in a patient with inactive rheumatoid arthritis. J Clin Rheumatol 2012;18:33-35.

16. Sheikh RA, Prindiville TP, Pecha RE, Ruebner BH. Unusual presentations of eosinophilic gastroenteritis: case series and review of literature. World J Gastroenterol 2009;15:21562161.

17. Farani JB, Albuquerque CB, de Oliveira JM, de Assis EA, de Oliveira Ayres Pinto E, de Lacerda Bonfante H. Arthritis, eosinophilia, and autoimmune liver disease. J Clin Rheumatol 2015;21:95-98.

18. Ng WF, Cohen P, Hepburn A, Hamdulay S, Carpani M, Mason JC. A case of eosinophilic enteritis and rheumatoid arthritis. Rheumatology 2005;44:1585-1586.

19. Chaudhuri K, Dubey S, Zaphiropoulos G. Idiopathic hypereosinophilic syndrome in a patient with long-standing rheumatoid arthritis: a case report. Rheumatology 2002;41:349350. 